reported that, in the rat, the response of smooth muscles to adrenergic or cholinergic stimulation greatly increased after the withdrawal of chronic treatment with morphine-reaching a peak three days later.

In another direction, the mechanisms of dependence suggested some years ago require an interaction of morphine with a humoral mediator involved in the activity of neurones in the brain. In this direction, some setbacks and some encouraging findings were recorded. On the one hand, the evidence adduced by Drs A. Goldstein (Stanford University) and I. Marshall (St Mary's Hospital Medical School, London) was against the proposition, stoutly defended by Dr Way, that a change in the level or turnover of brain 5-hydroxytryptamine is important in morphine dependence in the mouse. On the other hand, C. B. Smith and J. E. Villarreal (University of Michigan, Ann Arbor) reported that continued treatment with morphine and other addictive opiates increases the incorporation of ${ }^{14} \mathrm{C}$-tyrosine into the noradrenaline and dopamine of the brain of the mouse. The increased turnover of brain catecholamines can not be induced by strong antagonists of morphine and is blocked by naloxone ; in the author's opinion it is correlated with addictive liability in this group of drugs.

\section{BACTERIOLOGY}

\section{More Pollution}

from a Correspondent

THE summer conference of the Society for Applied Bacteriology was held at the University of Liverpool from July 13 to 15 and took the form of a symposium on the microbial aspects of pollution. The contributions and subsequent discussions interpreted the title in the broadest possible terms so that well discussed topics, such as the pollution of the aquatic environment, and lesser known forms of pollution, such as the disposal of infected laboratory materials, were included. Few new data were presented, but they illustrated the importance of microbiological processes in the armoury for combating today's pollution problems.

Dr A. L. Downing (Water Pollution Research Laboratory, Stevenage) warned microbiologists not to get too excited at the prospect of an increased number of jobs for their profession in this expanding field because the introduction of new and more stringent existing standards for effluent quality could well result in non-biological methods becoming increasingly more important.

Problems pertaining to the eutrophication of surface waters were covered in detail by four speakers. Professor P. L. McCarty and Dr R. T. Haug (Stanford University, California) discussed the various methods available for the removal of nitrogen from sewage effluents by the microbiological processes of nitrification and denitrification and presented evidence for supporting the use of aerobic and anaerobic upward-flow submerged filters. Dr N. P. Burman (Metropolitan Water Board, London) pointed out, however, that the removal of nitrogen and phosphorus from sewage effluents was not always the answer to eutrophication problems and gave the example of the Thames valley reservoirs where eutrophic conditions were inevitable because sufficient quantities of these elements were available from sources other than sewage.

Aerobic and anaerobic biological treatment of sewage and sewage sludges was discussed from both the microbiological and applied points of view and it was particularly encouraging to hear that the lead given by the deter- gents industry which changed from the production of biologically "hard" to "soft" materials is seriously being considered by industries producing other widely used materials. First, Dr H. O. W. Eggins (University of Aston) spoke on the possibilities of the plastics industry producing materials with parts in the chemical chain susceptible to microbial attack which would cause depolymerization and allow the subsequent attack of the smaller molecules by other microbes. Second, Dr S. L. J. Wright (Bath University of Technology) stated that a particularly attractive idea concerning the degradation of herbicides was the possibility of spraying the treated soil with an appropriate active microbial culture after an optimum time; preliminary experiments had already met with success. Third, Dr R. E. Cripps (Shell Research Ltd, Sittingbourne) spoke of his work on the relation between the chemical structure of pesticides and the possibilities of their degradation by microbial populations.

\title{
Chromosome Replication in Two Directions
}

For the double stranded and covalently closed circular DNA molecule which constitutes the chromosome of Escherichia coli to replicate in a semiconservative fashion the two parental strands of DNA must separate. There is, of course, evidence indicating that this separation is achieved by breaking phosphodiester bonds of the backbone of one of the two strands such that the nicked strand can be unwound from the circular strand. (Whether a single nick suffices or the chromosome is repeatedly nicked during replication is in the long run immaterial, for the net result is the same.) Moreover, genetic experiments have shown that replication begins and proceeds sequentially from a fixed point on the chromosome, the origin of replication. In theory at least replication might proceed in one or in both directions from this origin and until now it has generally been assumed that replication is unidirectional, proceeding $360^{\circ}$ from the origin back to it. In next Wednesday's Nature New Biology, however, Masters and Broda challenge this notion; their experiments lead to the conclusion that replication occurs in both directions from the origin.

Masters and Broda have, in essence, measured the frequency of occurence of particular genetic markers situated at either side of the origin of replication in cultures of cells growing at different rates. They used the generalized transducing phage P1 which picks up $E$. coli genes at particular frequencies dependent on the number of copies of the genes present in the cell and the ease with which they can be integrated into the phage genome.

Ratios of the frequency of particular genetic markers measured in this way fall on a two directional gradient when plotted against the markers' genetic map positions. Moreover, a series of elegant transduction experiments with various male and female strains of $E$. coli indicate that the relative frequency of transduction of a particular marker depends on the position of that marker in the chromosome.

In dividing cells it seems that there are more copies of markers immediately adjacent to both sides of the origin of replication than there are copies of genes distal from the origin and genes close to the origin are more often transduced than genes distal from it. Both these findings indicate that replication proceeds in both directions from a fixed origin. Furthermore, assuming that the current genetic map accurately represents the length of DNA between markers, it seems that the rate of replication in the two directions is not the same. The terminus of replication is not $180^{\circ}$ from the origin and replication in a clockwise direction about the chromosome seems to be faster than replication in the counterclockwise direction. In other words, Masters and Broda claim that replication of the $E$. coli chromosome is bidirectional but asymmetric and, as they note, Caro and his colleagues have reached the same conolusion from quite different experiments. 